APJ SUPPLEMENT SERIES, IN PRESS

Preprint typeset using LTEX style emulateapj v. 08/22/09

\title{
THE PERIOD-LUMINOSITY RELATION OF RR LYRAE STARS IN THE SDSS PHOTOMETRIC SYSTEM
}

\author{
C. CÁCEREs \& M. CATElan \\ Pontificia Universidad Católica de Chile, Departamento de Astronomía y Astrofísica, \\ Av. Vicuña Mackenna 4860, 782-0436 Macul, Santiago, Chile; \\ ApJ Supplement Series, in press
}

\begin{abstract}
We provide the first detailed study of the RR Lyrae period-luminosity (PL) relation in the ugriz bandpasses of the Sloan Digital Sky Survey (SDSS) filter system. We argue that tight, simple PL relations are not present in the SDSS filters, except for the redder bandpasses $i$ and (especially) $z$. However, for all bandpasses, we show that, by incorporating terms involving a (fairly reddening-independent) "pseudo-color" $C_{0} \equiv(u-g)_{0}-(g-r)_{0}$, tight (non-linear) relations do obtain. We provide theoretically calibrated such relations in the present paper, which should be useful to derive precise absolute magnitudes (hence distances) and intrinsic colors (hence reddening values) to even individual field RR Lyrae stars. For applications to cases where photometry in all five passbands may not be available, we also provide simple (though less precise) average PL relations for the $i$ and $z$ bandpasses, which read as follows:

$$
\begin{aligned}
& M_{z}=0.839-1.295 \log P+0.211 \log Z, \\
& M_{i}=0.908-1.035 \log P+0.220 \log Z .
\end{aligned}
$$
\end{abstract}

Similarly, simple period-color relations for $(r-i)_{0},(g-r)_{0}$, and $(u-z)_{0}$ are also provided.

Subject headings: stars: distances — stars: horizontal-branch — stars: variables: other — distance scale

\section{INTRODUCTION}

The Sloan Digital Sky Survey (SDSS) has given the scientific comunity an unprecedented chance to systematically map a large area of the sky, using its own special 5-band filter system. In the process, an overwhelming amount of data has been ammassed, which can be used to perform many different types of scientific studies. Of particular interest to us is the fact that the SDSS has also provided, with unprecedented detail, a map of the spatial distribution of different stellar populations accross the Galaxy, which is increasingly being used to trace new structures in the Galactic halo (e.g., Belokurov et al. 2006), some of which may plausibly be related to the hundreds of elusive "protogalactic fragments" that are predicted, in the $\Lambda \mathrm{CDM}$ cosmological paradigm, to have given birth to a Galaxy like the Milky Way (e.g., Abadi et al. 2003). In this sense, RR Lyrae stars have proven to be a stellar component that is consistently present in most, and possibly all, of these structures (e.g., Greco et al. 2008; Kuehn et al. 2008). Light curves for many variable stars in the SDSS filter system have been provided by the SDSS II Survey, a 3-year extension of the original SDSS survey, with images of the same fields taken every other night, with the main goal to detect supernovae explosions. However, it remains at present difficult to obtain reliable distances therefrom, since so far no detailed study of the properties of RR Lyrae stars in the SDSS system has been performed, with the notable exception of Marconi et al. (2006). In the same vein, it is still not possible at present to directly extract reliable distance information from detailed RR Lyrae light curves obtained in the SDSS system, as are now becoming increasingly common (e.g., De Lee, Smith, \& Beers 2007; Sesar et al. 2007; Wilhelm et al. 2008).

Accordingly, the purpose of the present study is to provide the first systematic investigation of the RR Lyrae periodluminosity (PL) relation in the bandpasses of the SDSS system, which should enable the determination of more reli- able distances to individual RR Lyrae stars for which data in the SDSS system are available than has been possible thus far. This paper presents an extension of the work by Catelan, Pritzl, \& Smith (2004), who obtained such a PL relation for the $U B V R I J H K$ passbands of the JohnsonCousins-Glass photometric system, and by Cortés \& Catelan (2008), who studied the PL relations in the Strömgren system. Its structure is quite similar to that in Catelan et al. and Cortés \& Catelan. We begin by presenting, in $\$ 2$, the theoretical framework upon which our study is based. In $\$ 3$, we explain the origin of the derived PL relations, whose calibrations are provided in $\$ 4$. Some final remarks are provided in $\S 6$.

\section{MODELS}

In order to derive the PL relations, we computed a series of horizontal branch (HB) simulations, following recipes similar to those presented in Catelan et al. (2004) and Cortés \& Catelan (2008). We used the evolutionary tracks given by Sweigart \& Catelan (1998) and Catelan et al. (1998) for $Z=0.0005,0.001,0.002$, and 0.006. The main-sequence helium abundance by mass is assumed to be $Y=0.23$, and solar-scaled compositions are adopted. Catelan et al. argue that these models are consistent with a distance modulus to the Large Magellanic Cloud of $(m-M)_{0}=18.47 \mathrm{mag}$. Note, however, that this value is based on the empirical prescriptions for the LMC by Gratton et al. (2004); using the independent measurements by Borissova et al. (2004), a distance modulus of $(m-M)_{0}=18.50$ mag would derive instead.

The mass distribution is assumed to be a normal deviate, with a mass dispersion of $\sigma_{M}=0.02 M_{\odot}$. In order to pass from the theoretical $\left(\log L, \log T_{\text {eff }}\right)$ plane to the empirical ones in which the magnitudes in the SDSS photometric system $(u, g, r, i$, and $z)$ are used, we have incorporated the bolometric corrections from Girardi et al. (2004) to the 

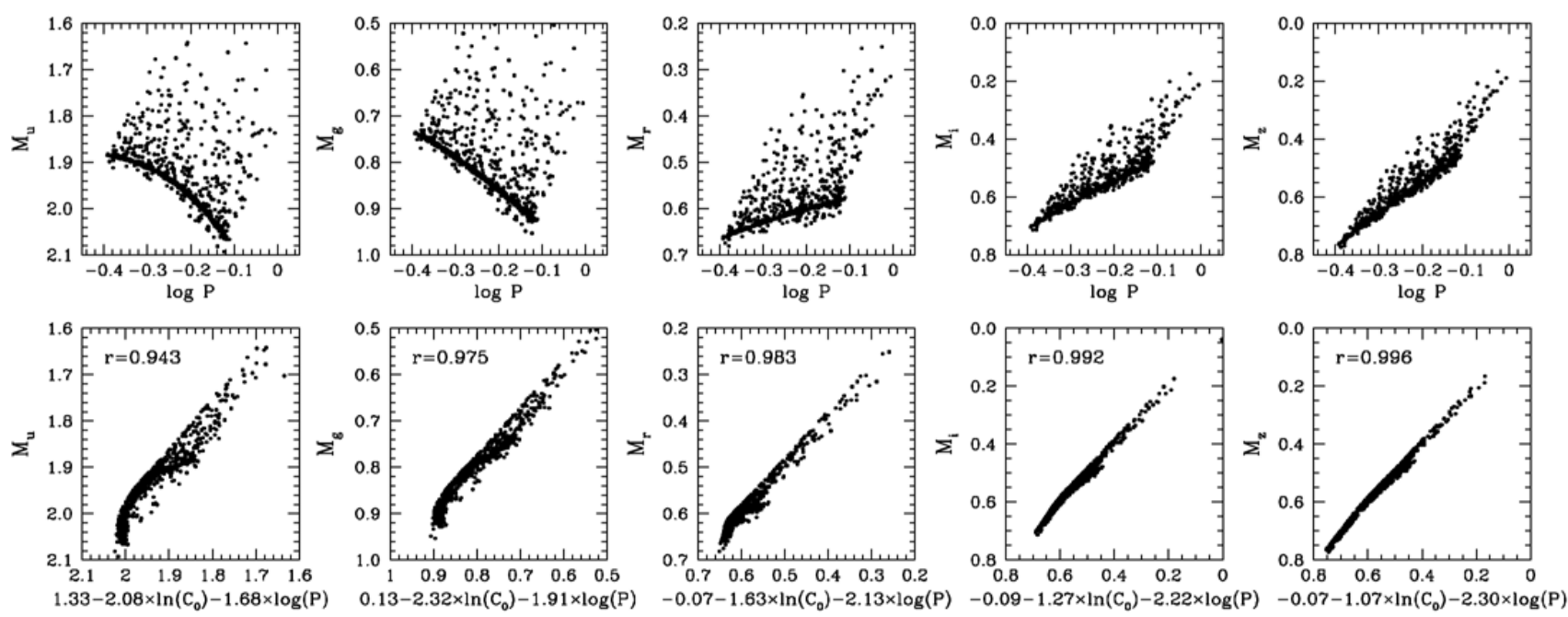

FIG. 1.- Upper panels: RR Lyrae PL relations for the different indicated SDSS filters. Lower panels: Corresponding RR Lyrae distributions in the absolute magnitude-log-period-(pseudo-)color plane. Note the dramatic reduction in scatter that is brought about with the inclusion of a $C_{0}$-dependent term (the correlation coefficient $r$ is shown in the lower panels). All plots show 650 randomly chosen synthetic RR Lyrae stars from an HB simulation with $Z=0.002$ and an intermediate HB type.
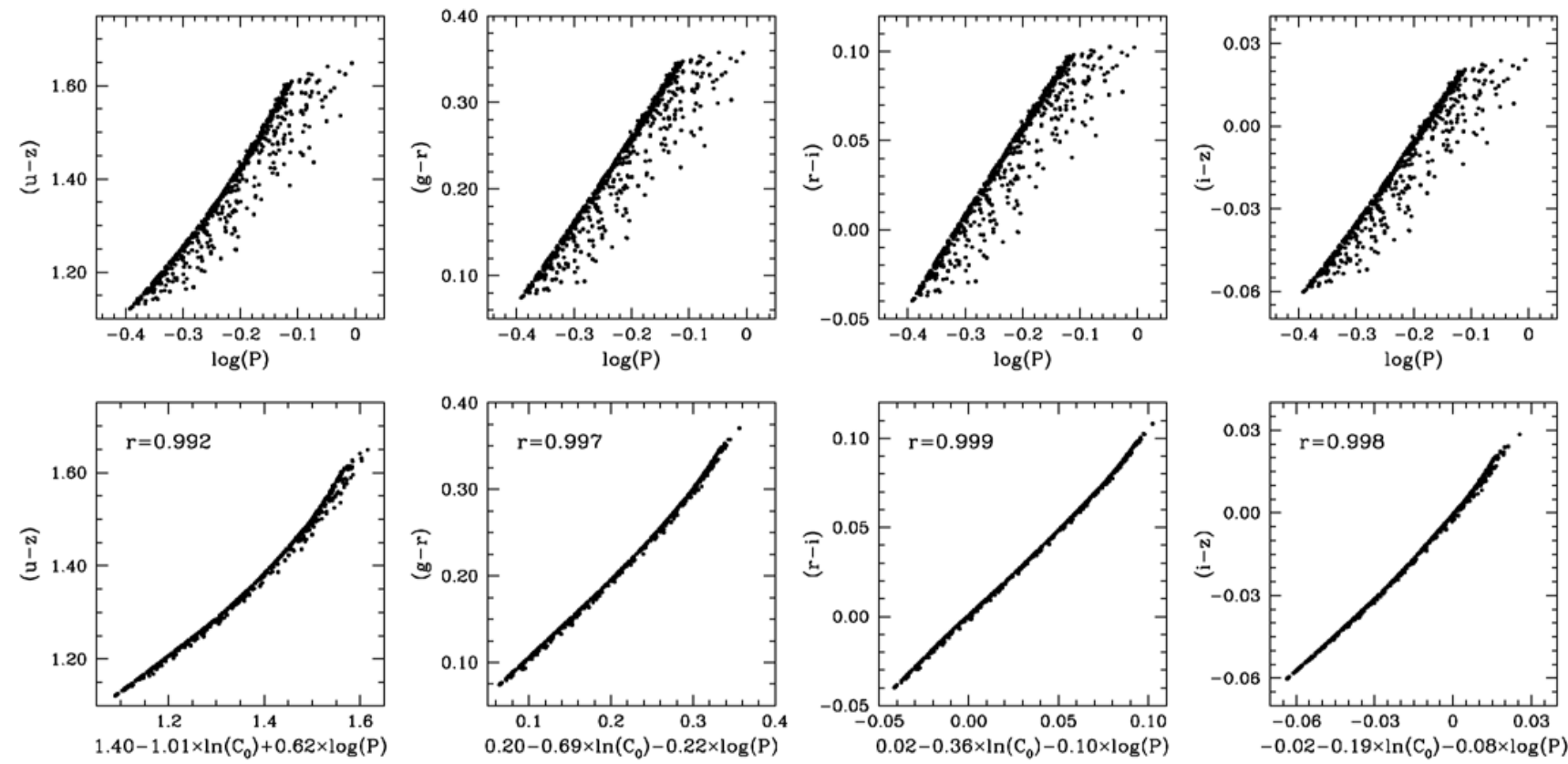

FIG. 2.- Upper panels: RR Lyrae PC relations for the $(u-z),(g-r),(r-i)$ and $(i-z)$ SDSS-based colors. Lower panels: Corresponding RR Lyrae distributions in the color-log-period-(pseudo-)color plane. Note the dramatic reduction in scatter that is brought about with the inclusion of a $C_{0}$-dependent term (the correlation coefficient $r$ is shown in the lower panels). All plots show 650 randomly chosen synthetic RR Lyrae stars from an HB simulation with $Z=0.002$ and an intermediate HB type.

code, over the relevant ranges of temperature and gravity. The blue edge of the instability strip is computed as prescribed by Caputo et al. (1987), but with a shift of $-200 \mathrm{~K}$ applied to the resulting temperatures to provide better agreement with recent prescriptions (Catelan 2004). The instability strip width is taken as $\Delta \log T_{\text {eff }}=0.075$, which gives us the temperature of the red edge of the instability strip once the blue edge position has been found. When a star lies between the blue and red edges, its period is calculated based on equation (4) of Caputo et al. (1998). Therefore, our relations are directly applicable to fundamental-mode (i.e., RRab or RR0) stars, but the observed periods of first-overtone (RRc or RR1) stars must first be "fundamentalized" according to the relation $\log P_{f}=\log P_{c}+0.128$ (Catelan 2005, and references therein) before comparing with our results.

In this paper we are interested in finding PL relations for RR Lyrae stars in the SDSS system. To properly take into account the impact of variations in HB morphology, for each of the four studied metallicities we computed extensive series of $\mathrm{HB}$ simulations, including $\mathrm{HB}$ types that range from very red to very blue. This leads to a total of 423,766 synthetic RR Lyrae stars that cover a wide range in metallicity and HB types. These stars are then used to search for possible PL relations, as described in the next sections.

\section{GENESIS OF THE PL RELATIONS IN THE SDSS SYSTEM}


TABLE 1

COEFFICIENTS OF THE FITS

\begin{tabular}{|c|c|c|c|c|c|}
\hline Coefficient & Value & Error & Coefficient & Value & Error \\
\hline \multicolumn{3}{|c|}{$z$} & \multicolumn{3}{|c|}{$(u-g)_{0}$} \\
\hline$a_{0}$ & 1.3706 & 0.0083 & $a_{0}$ & 2.1983 & 0.0030 \\
\hline$a_{1}$ & 0.8941 & 0.0061 & $a_{1}$ & 0.6282 & 0.0022 \\
\hline$a_{2}$ & 0.1315 & 0.0011 & $a_{2}$ & 0.0939 & 0.0004 \\
\hline$b_{0}$ & -2.6907 & 0.0372 & $b_{0}$ & -1.3677 & 0.0133 \\
\hline$b_{1}$ & -0.8192 & 0.0272 & $b_{1}$ & -1.0556 & 0.0097 \\
\hline$b_{2}$ & -0.0664 & 0.0049 & $b_{2}$ & -0.1537 & 0.0018 \\
\hline$c_{0}$ & 47.9836 & 0.3659 & $c_{0}$ & 36.8370 & 0.1311 \\
\hline$c_{1}$ & 31.7879 & 0.2676 & $c_{1}$ & 24.1751 & 0.0959 \\
\hline$c_{2}$ & 5.2221 & 0.0480 & $c_{2}$ & 3.9662 & 0.0172 \\
\hline$d_{0}$ & 141.7704 & 1.2837 & $d_{0}$ & 114.0780 & 0.4599 \\
\hline$d_{1}$ & 100.6676 & 0.8919 & $d_{1}$ & 80.9562 & 0.3195 \\
\hline$d_{2}$ & 17.4277 & 0.1541 & $d_{2}$ & 13.9697 & 0.0552 \\
\hline$e_{0}$ & 0.3286 & 0.0254 & $e_{0}$ & 1.8627 & 0.0091 \\
\hline$e_{1}$ & 2.0377 & 0.0186 & $e_{1}$ & 1.1096 & 0.0067 \\
\hline$e_{2}$ & 0.3882 & 0.0034 & $e_{2}$ & 0.1791 & 0.0012 \\
\hline \multicolumn{3}{|c|}{$(g-r)_{0}$} & \multicolumn{3}{|c|}{$\left(r-i_{0}\right)$} \\
\hline$\overline{a_{0}}$ & 1.1983 & 0.0030 & $a_{0}$ & 0.3218 & 0.0011 \\
\hline$a_{1}$ & 0.6282 & 0.0022 & $a_{1}$ & 0.1907 & 0.0008 \\
\hline$a_{2}$ & 0.0939 & 0.0004 & $a_{2}$ & 0.0290 & 0.0001 \\
\hline$b_{0}$ & -2.3672 & 0.0133 & $b_{0}$ & -0.8054 & 0.0049 \\
\hline$b_{1}$ & -1.0552 & 0.0097 & $b_{1}$ & -0.2516 & 0.0036 \\
\hline$b_{2}$ & -0.1536 & 0.0018 & $b_{2}$ & -0.0313 & 0.0006 \\
\hline$c_{0}$ & 36.3361 & 0.1311 & $c_{0}$ & 15.8088 & 0.0487 \\
\hline$c_{1}$ & 24.1760 & 0.0959 & $c_{1}$ & 10.8036 & 0.0356 \\
\hline$c_{2}$ & 3.9668 & 0.0172 & $c_{2}$ & 1.8125 & 0.0064 \\
\hline$d_{0}$ & 113.8830 & 0.4599 & $d_{0}$ & 46.0807 & 0.1708 \\
\hline$d_{1}$ & 80.9365 & 0.3195 & $d_{1}$ & 33.1513 & 0.1187 \\
\hline$d_{2}$ & 13.9681 & 0.0552 & $d_{2}$ & 5.7868 & 0.0205 \\
\hline$e_{0}$ & 1.8627 & 0.0091 & $e_{0}$ & 0.7327 & 0.0034 \\
\hline$e_{1}$ & 1.1097 & 0.0067 & $e_{1}$ & 0.4298 & 0.0025 \\
\hline$e_{2}$ & 0.1792 & 0.0012 & $e_{2}$ & 0.0700 & 0.0004 \\
\hline \multicolumn{3}{|c|}{$(i-z)_{0}$} & & & \\
\hline$\overline{a_{0}}$ & 0.2050 & 0.0007 & & & \\
\hline$a_{1}$ & 0.1497 & 0.0005 & & & \\
\hline$a_{2}$ & 0.0242 & 9.6205 & & & \\
\hline$b_{0}$ & -0.7589 & 0.0033 & & & \\
\hline$b_{1}$ & -0.3685 & 0.0024 & & & \\
\hline$b_{2}$ & -0.0559 & 0.0004 & & & \\
\hline$c_{0}$ & 8.7812 & 0.0320 & & & \\
\hline$c_{1}$ & 5.7009 & 0.0234 & & & \\
\hline$c_{2}$ & 0.9193 & 0.0042 & & & \\
\hline$\widetilde{d_{0}}$ & 32.0594 & 0.1123 & & & \\
\hline$d_{1}$ & 22.1549 & 0.0781 & & & \\
\hline$d_{2}$ & 3.7400 & 0.0135 & & & \\
\hline$e_{0}$ & 0.4759 & 0.0022 & & & \\
\hline$e_{1}$ & 0.2728 & 0.0016 & & & \\
\hline$e_{2}$ & 0.0446 & 0.0003 & & & \\
\hline
\end{tabular}

TABLE 2

ANALYTICAL FITS QUALITY DIAGNOSTICS ${ }^{1}$

\begin{tabular}{lcc}
\hline \hline \multicolumn{1}{c}{ Fit } & $r$ & std. error \\
\hline$z$ & 0.9981 & 0.0096 \\
$(u-g)$ & 0.9974 & 0.0034 \\
$(g-r)$ & 0.9991 & 0.0034 \\
$(r-i)$ & 0.9995 & 0.0013 \\
$(i-z)$ & 0.9994 & 0.0008
\end{tabular}

1 For the fits given by equation (3) and Table 1 .
As discussed in Catelan et al. (2004), the expected PL relation must be tighter towards the redder passbands (especially in the near-infrared), compared to the visual bands. The effects of temperature and luminosity on the periods affect strongly the shape of the resulting PL relation. In order to better appreciate this, recall that, from the period-mean density relation or Ritter's relation (e.g., van Albada \& Baker 1971), periods increase strongly with both an increase in luminosity and a decrease in temperature. While the luminosities of RR Lyrae stars are remarkably uniform for a given metallicity and HB type, the introduction of filters, with their often strongly temperature-dependent bolometric corrections, may add strong slopes to the otherwise "horizontal" branch. Thus in $u$ and $g$, the cooler stars appear fainter than the bluer ones; conversely, in the redder passbands ( $r, i$, and $z$ ), the cooler stars are the ones that appear brighter. Since the cooler/more luminous stars are the ones with longer periods, the end result is that the PL relation will appear increasingly tighter towards the redder passbands, the inverse happening towards the bluer passbands (see Catelan et al. 2004, for a detailed discussion).

This behavior is confirmed in Figure 1 (top row), where we show the changes in the absolute magnitude-log-period space, for $\mathrm{HB}$ simulations computed for a rather even $\mathrm{HB}$ morphology, a metallicity of $Z=0.002$, and each of the ugriz SDSS passbands. Qualitatively similar results are obtained for other metallicity values and HB morphologies as well. As can be clearly seen, it is only when the redder bandpasses of the SDSS sytem - namely, $i$ and $z$ - are used that one begins to find relatively tight, simple PL relations. This behavior is in total agreement with the previous results by Catelan et al. (2004), who had similarly found that, in the case of the Johnson-Cousins-Glass system, such simple PL relations are present only for $I$ and redder bandpasses.

\section{THE RR LYRAE PL RELATION IN THE SDSS SYSTEM CALIBRATED}

\subsection{Relations Involving a "Pseudo-Color"}

As shown in Cortés \& Catelan (2008), the originally very poor PL relations in the Strömgren (1963) filter system become exceedingly tight when (fairly reddening-independent) Strömgren "pseudo-color" $C_{0} \equiv(u-v)_{0}-(v-b)_{0}$ terms are incorporated into these relations. Can something similar be accomplished, in the case of the SDSS system?

To answer this question, we have searched for a combination of blue and red SDSS colors that might also prove relatively reddening-free. We used the extinctions provided online by $D$. Schlegel, ${ }^{1}$ according to which one has $E(u) / E(B-V)=5.16, E(g) / E(B-V)=3.79, E(r) / E(B-V)=$ $2.75, E(i) / E(B-V)=2.09$, and $E(z) / E(B-V)=1.48$. On this basis, we find that a "pseudo-color" defined as

$$
C_{0}=(u-g)_{0}-(g-r)_{0}
$$

turns out to be fairly reddening-insensitive, with the unreddened $\left(C_{0}\right)$ and reddened $\left(C_{1}\right)$ quantities being related by the following equation:

$$
C_{0}=C_{1}-0.32 E(B-V) \text {. }
$$

That by incorporating such $C_{0}$-dependent terms can indeed lead to much tighter PL-pseudo-color (PLpsC) relations is 
confirmed by Figure 1 (bottom row), which shows the enormous improvement over the situation in which no such terms are included (Fig. 1, top row).

In the course of our research we noticed that tight periodcolor-pseudo-color (PCpsC) relations also obtain, when one includes such $C_{0}$-dependent terms. This is clearly shown in Figure $2{ }^{2}$ As a matter of fact, such relations are even tighter than the corresponding PLpsC ones. Therefore, in what follows, we shall directly provide our theoretically calibrated PLpsC relation in a single bandpass, namely $z$ (which provides us with the highest correlation coefficient of all the SDSS filters), electing to provide PCpsC relations involving the remainder of the SDSS filters, due to their higher correlation coefficients. From the provided PLpsC relation in $z$ and the PCpsC relations, one can straightforwardly derive PLpsC relations for all other individual SDSS bandpasses.

The final relations that we obtained are thus of the form:

$$
\begin{aligned}
\text { mag or color }=\sum_{i=0}^{2} a_{i}(\log Z)^{i} & +\sum_{i=0}^{2} b_{i}(\log Z)^{i}\left(\ln C_{0}\right) \\
& +\sum_{i=0}^{2} c_{i}(\log Z)^{i}\left(\ln C_{0}\right)^{2} \\
& +\sum_{i=0}^{2} d_{i}(\log Z)^{i}\left(\ln C_{0}\right)^{3} \\
& +\sum_{i=0}^{2} e_{i}(\log Z)^{i}(\log P),
\end{aligned}
$$

where "mag" stands for the absolute magnitude in $z$, whereas "color" represents any of the colors $(u-g)_{0},(g-r)_{0},(r-i)_{0}$, and $(i-z)_{0}$. In this expression, $C_{0}$ is the SDSS system's pseudo-color [eq. (1)], and $P$ is the fundamentalized RR Lyrae period (in days). The corresponding coefficients, along with their errors, are given in Table 1 . (Naturally, the $c_{0}$ coefficient that appears in this table is not the same as the pseudo-color $C_{0}$, which is given in capital letters throughout this paper to avoid confusion.)

We stress that these equations are able to reproduce the input values (from the HB simulations) with high precision. This is shown in Table 2, where the correlation coefficient $r$ and the standard error of the estimate are given for each of the four equations. We also show, in Figures 3, 4, 5, 6, and 7] the residuals [in the sense eq. (3) minus input values (from the simulations)] for a random subset of 6000 synthetic stars drawn from the original pool of 423,766 synthetic RR Lyrae stars in the HB simulations, for the fits computed for $z$, $(u-g)_{0},(g-r)_{0},(r-i)_{0}$, and $(i-z)_{0}$, respectively. These plots further illustrate that the SDSS magnitudes and colors can be predicted from the data provided in equation (3) and Table 1 with a precision that is generally at the level of $0.01 \mathrm{mag}$ (or better, especially at low metallicities).

Finally, we note that equation (3) can be trivially expressed in terms of $[\mathrm{Fe} / \mathrm{H}]$; this can be accomplished using the relation

$$
\log Z=[\mathrm{M} / \mathrm{H}]-1.765,
$$

\footnotetext{
${ }^{2}$ Note that here we prefer to provide a plot for the $(u-z)_{0}$ color as opposed to $(u-g)_{0}$, since we have found that the latter presents a complex, non-linear behavior as a function of $\ln C_{0}$ and $\log P$. This is also the reason why, in equation 10 below, we provide a simple linar fit for $(u-z)_{0}$, rather than $(u-$ $g)_{0}$, as a function of $\log P$ and $\log Z$.
}

which is the same as equation (9) in Catelan et al. (2004). In this sense, the effects of an enhancement in $\alpha$-capture elements with respect to a solar-scaled mixture, such as observed amongst Galactic halo stars (e.g., Pritzl, Venn, \& Irwin 2005, and references therein), can be taken into account by using the following scaling relation (Salaris, Chieffi, \& Straniero 1993):

$$
[\mathrm{M} / \mathrm{H}]=[\mathrm{Fe} / \mathrm{H}]+\log (0.638 f+0.362),
$$

where $f=10^{[\alpha / \mathrm{Fe}]}$. However, such a relation should be used with due care for metallicities $Z>0.003$ (VandenBerg et al. 2000).

\subsection{The Effect of the Helium Abundance}

The dependence of the RR Lyrae PLpsC relation in the SDSS system on the adopted width of the mass distribution, as well as on the helium abundance, has been analyzed by computing additional sets of synthetic HB's for $\sigma_{M}=0.030 M_{\odot}(Z=0.001)$ and for a main-sequence helium abundance of $28 \%(Z=0.002)$. The effect of $\sigma_{M}$ variations was found to be negligible (see also Catelan et al. 2004; Cortés \& Catelan 2008, for similar results in the case of the Johnson-Cousins-Glass and Strömgren systems, respectively), but helium turned out to have a more important influence (again as previously found in the other filter systems). The results are shown in Figures 8 (PLpsC relation in $z$ ) and 9(PLpsC relations).

In the case of $M_{z}$, there is a clear offset in the zero point, as well as an increased dispersion in comparison with Figure 8 Still, the standard deviation remains a modest 0.024 mag to be compared with the actual dispersion in $M_{z}$ magnitudes from the HB simulations for a fixed $Y$, which amounts to a full 1.25 mag (i.e., $M_{z}$ values for individual RR Lyrae stars range from $0.97 \mathrm{mag}$ at their faintest to $-0.29 \mathrm{mag}$ at their brightest), in the $Y_{\mathrm{MS}}=0.23$ case. Therefore, if a correction to the zero point $a_{0}$ for $M_{z}$ in Table 1 [and also in eq. (6) below] by $d M_{z} / d Y=-0.044 /(0.28-0.23)=-0.88$ [i.e., in the sense that eq. (3) predicts too faint magnitudes] is duly taken into account, equation (3) [and similarly eq. (6) below] can also be used to provide useful information on the absolute magnitudes of RR Lyrae stars with enhanced helium abundances.

\subsection{Simple Relations}

As in Catelan et al. (2004), and for the bandpasses that show sufficiently tight PL relations (i.e., $i$ and $z$; see Fig. 1), we have computed average PL relations that do not show an explicit dependence on $C_{0}$. The goal here is to enable an application of our derived PL relations even when observations in the bluer passbands of the SDSS system are not available. We do provide, however, simple relations for colors involving such bluer bandpasses. The resulting relations are as follows:

$$
M_{z}=0.839-1.295 \log P+0.211 \log Z,
$$

with a correlation coefficient $r=0.97$ and a standard error of the estimate of $0.037 \mathrm{mag}$;

$$
M_{i}=0.908-1.035 \log P+0.220 \log Z,
$$

with a correlation coefficient $r=0.95$ and a standard error of the estimate of $0.045 \mathrm{mag}$;

$$
(r-i)_{0}=0.184+0.438 \log P+0.017 \log Z,
$$




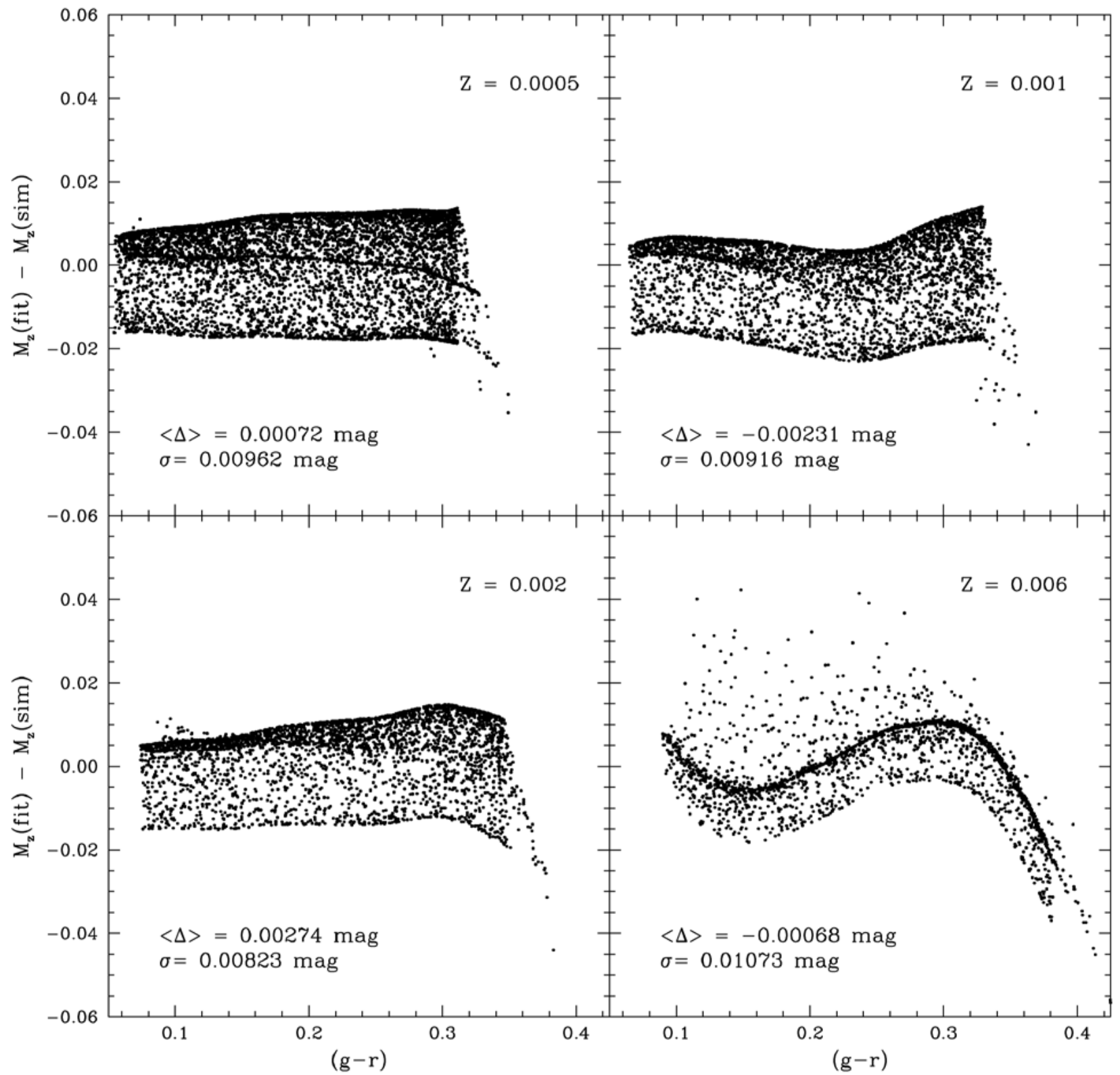

FIG. 3.- Difference between the absolute magnitude in $z$, as predicted by equation (3), and its input value (from the HB simulations), plotted as a function of the $(g-r)_{0}$ color (from the same simulations) for four different metallicities: $Z=0.0005$ (upper left), 0.001 (upper right), 0.002 (lower left), 0.006 (lower right). A total of 6000 randomly selected synthetic stars is shown for all metallicities. The average magnitude difference is indicated in the lower left corner of each panel, along with the corresponding standard deviation. The latter is a direct indicator of the precision with which equation 3 is able to provide the $M_{z}$ values.

with a correlation coefficient $r=0.95$ and a standard error of the estimate of $0.013 \mathrm{mag}$;

$$
(g-r)_{0}=0.640+0.851 \log P+0.081 \log Z,
$$

with a correlation coefficient $r=0.95$ and a standard error of the estimate of $0.027 \mathrm{mag}$; and

$$
(u-z)_{0}=2.317+1.472 \log P+0.221 \log Z,
$$

with a correlation coefficient $r=0.95$ and a standard error of the estimate of $0.045 \mathrm{mag}$. Note that, as a consequence of the large number of stars involved in the fits, the errors in all of the derived coefficient are very small (of order $10^{-4}-10^{-3}$ ). We performed tests in which quadratic terms were added to these equations, but in no case was the improvement of ma- jor significance, the standard errors of the estimates generally changing only in the third decimal place.

\subsection{On Applying Our Relations to RR Lyrae Stars}

When applying our equation (3) in globular cluster work, the metallicity of the cluster will often be known a priori. However, metallicity estimates may also be unavailable, especially when dealing with field RR Lyrae stars. Yet, for a reliable application of our relations to field stars, an estimate of their metallicities must be provided.

The SDSS system itself may itself come to our rescue in such a case. We recall that estimates of the RR Lyrae metallicities can be obtained on the basis of their $V$-band light curves using Fourier decomposition (e.g., Jurcsik \& Kovács 1996; Jurcsik 1998; Kovács \& Kupi 2007; Morgan. Wahl, \& Wieckhorst 2007). Transforma- 


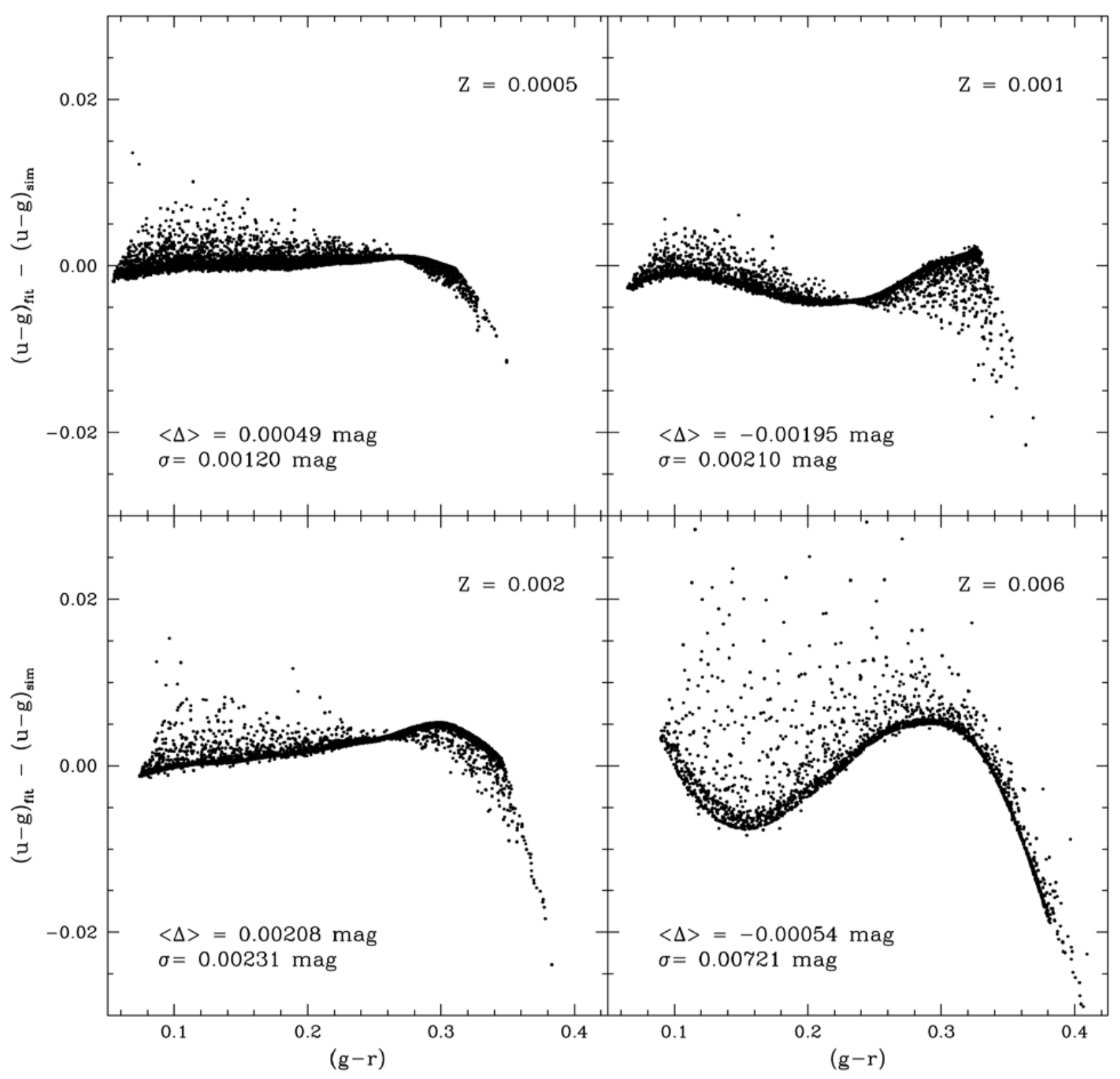

FIG. 4.- As in Figure 3 but for $(u-g)_{0}$.

tion equations between the SDSS system and the JohnsonCousins system have been provided in the literature (e.g., Karaali, Bilir, \& Tunçel 2005), and an updated list of such transformation equations is maintained at the SDSS web site. ${ }^{3}$ For instance, from the current "Lupton set" one finds the following:

$$
V=g-0.2906(u-g)+0.0885,
$$

with a $\sigma=0.013 \mathrm{mag}$, and

$$
V=g-0.5784(g-r)-0.0038,
$$

with a $\sigma=0.005 \mathrm{mag}$. Thus, on the basis of $V$-band light curves computed from SDSS $u$ - and $g$ - (or, alternatively, $g$ and $r$-) band magnitudes, one should be able to estimate

\footnotetext{
3 http://www.sdss.org/dr4/algorithms/sdssUBVRITransform.html
}

metallicities for individual RR Lyrae stars, through Fourier decomposition. We note, in addition, that in a forthcoming paper (Catelan \& Cáceres 2008, in preparation) we will be providing analytical fits that should allow one to estimate metallicity values directly from the SDSS photometry.

The reader should be warned that our relations should be compared against empirical quantities obtained for the socalled equivalent static star. Several procedures have been advanced in the literature for the determination of the latter on the basis of empirically derived magnitudes and colors (e.g., Bono, Caputo, \& Stellingwerf 1995, and references therein). In particular, one should note that, according to the hydrodynamical models provided by Bono et al., one should expect differences between temperatures derived from intensityor magnitude-averaged colors, on the one hand, and those based on the actual color of the equivalent static star, on the other. As a workaround, these authors set forth very use- 


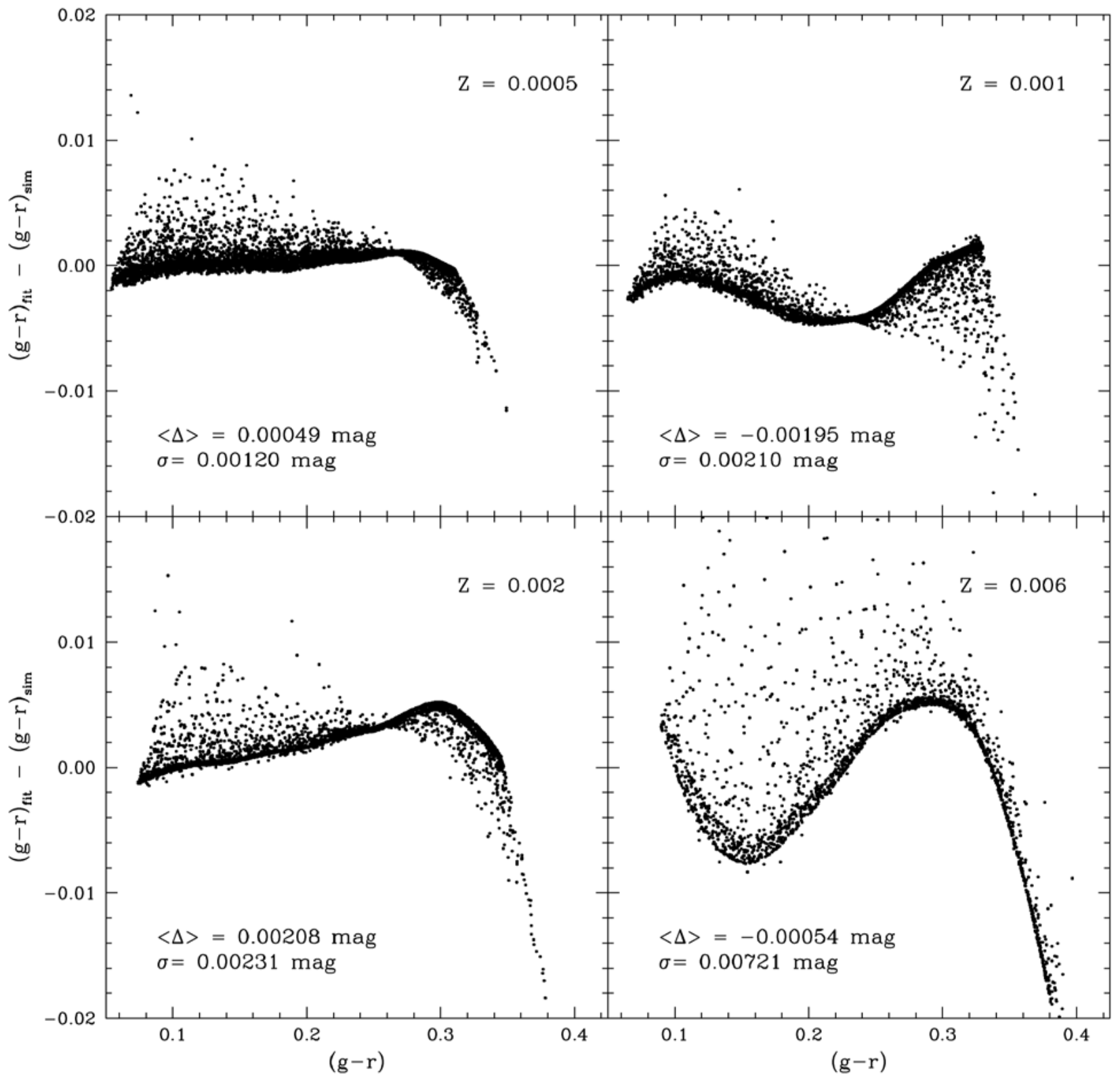

FIG. 5.- As in Figure 3 but for $(g-r)_{0}$.

ful amplitude-dependent corrections, which become more important the bluer the bandpass. More recently, Marconi et al. (2006) analyzed the problem in the specific case of the SDSS system, concluding that intensity averages, though not perfect, are to be preferred over averages carried out in magnitude units. Unfortunately, tables with amplitude-dependent corrections that would allow one to properly compute magnitudes and colors in the SDSS system for the equivalent static star (i.e., similar to those provided by Bono et al. 1995, in the Johnson-Cousins sytem) have not yet been provided in the literature. One way or another, the reader should note that $C_{0}$, being a difference between two colors, is presumably affected to a lesser degree than are the colors themselves (see also Cortés \& Catelan 2008). Needless to say, observers are also strongly warned against using single-epoch photometry to derive $C_{1}$ values to be used along with our relations.

\section{SUMMARY}

We have provided the first extensive calibration of the RR Lyrae PL (and PC) relations in the SDSS ugriz filter system. As in Catelan et al. (2004), we find that these PL relations become progressively tighter for the redder passbands, those in $i$ and $z$ appearing particularly promising. We provide very precise relations involving a newly defined, fairly reddening-insensitive pseudo-color $C_{0} \equiv(u-g)_{0}-(g-r)_{0} . C_{0^{-}}$ independent, though less precise, average relations are also provided for those cases in which observations in all five SDSS filters may not be available. Our relations should be especially useful for the calculation of distances and reddenings to even individual field RR Lyrae stars.

We thank H. A. Smith and N. De Lee for interesting discussions. The authors acknowledge financial support by Proyecto 


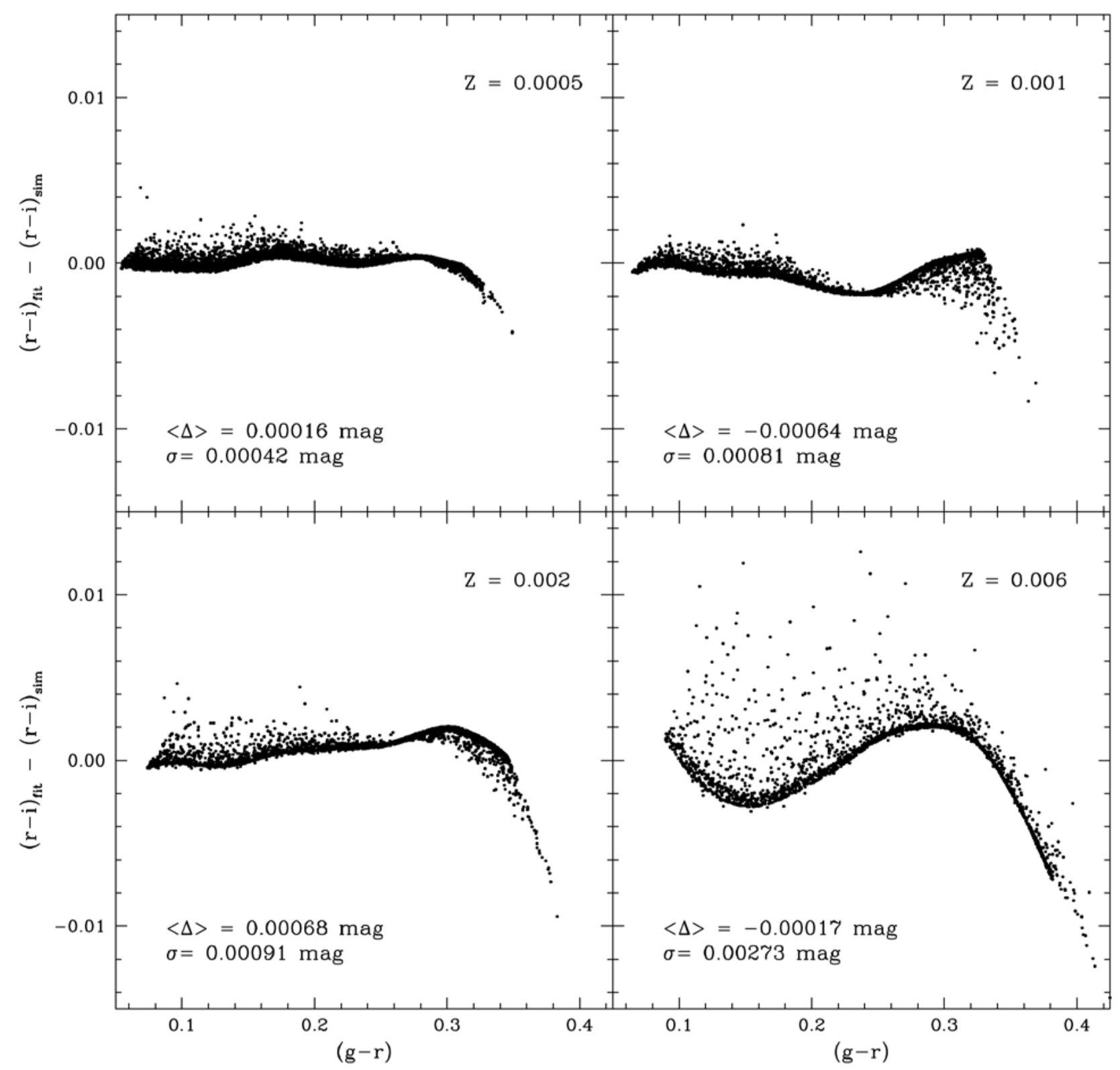

FIG. 6.- As in Figure 3 but for $(r-i)_{0}$.

FONDECYT Regular No. 1071002.

\section{REFERENCES}

Abadi, M. G., Navarro, J. F., Steinmetz, M., \& Eke, V. R. 2003, ApJ, 591, 499

Belokurov, V., et al. 2006, ApJ, 654, 897

Bono, G., Caputo, F., \& Stellingwerf, R. F. 1994, ApJ, 432, L51

Bono, G., Caputo, F., \& Stellingwerf, R. F. 1995, ApJS, 99, 263

Borissova, J., Minniti, D., Rejkuba, M., Alves, D., Cook, K. H., \& Freeman, K. C. 2004, A\&A, 423, 97

Caputo, F., De Stefanis, P., Paez, E., \& Quarta, M. L. 1987, A\&AS, 68, 119

Caputo, F., Marconi, M., \& Santolamazza, P. 1998, MNRAS, 293, 364

Catelan, M. 2004, ApJ, 600, 409

Catelan, M. 2005, preprint (astro-ph/0507464)

Catelan, M., Borissova, J., Sweigart, A. V., \& Spassova, N. 1998, ApJ, 494, 265

Catelan, M., Pritzl, B. J., \& Smith, H. A. 2004, ApJS, 154, 633

Cortés, C., \& Catelan, M. 2008, ApJS, in press (astro-ph/0802.2309)

De Lee, N. M., Smith, H. A., \& Beers, T. C. 2007, BAAS, 39, 211.2502
Girardi, L., Grebel, E. K., Odenkirchen, M., \& Chiosi, C. 2004, A\&A, 422, 205

Gratton, R. G., Bragaglia, A., Clementini, G., Carretta, E., Di Fabrizio, L., Maio, M., \& Taribello, E. 2004, A\&A, 421, 937

Greco, C., et al. 2008, ApJ, 675, L73

Jurcsik, J. 1998, A\&A, 333, 571

Jurcsik, J., \& Kovács, G. 1996, A\&A, 312, 111

Karaali, S., Bilir, S., \& Tunçel, S. 2005, PASA, 22, 24

Kovács, G., \& Kupi, G. 2007, A\&A, 462, 1007

Kuehn, C., et al. 2008, ApJ, 674, L81

Marconi, M., Cignoni, M., Di Criscienzo, M., Ripepi, V., Castelli, F.,

Musella, I., \& Ruoppo, A. 2006, MNRAS, 371, 1503

Morgan, S. M., Wahl, J. N., \& Wieckhorst, R. M. 2007, MNRAS, 374, 1421

Pritzl, B. J., Venn, K. A., \& Irwin, M. 2005, AJ, 130, 2140

Salaris, M., Chieffi, A., \& Straniero, O. 1993, ApJ, 414, 580

Sesar, B., et al. 2007, AJ, 134, 2236

Strömgren, B. 1963, QJRAS, 4, 8 


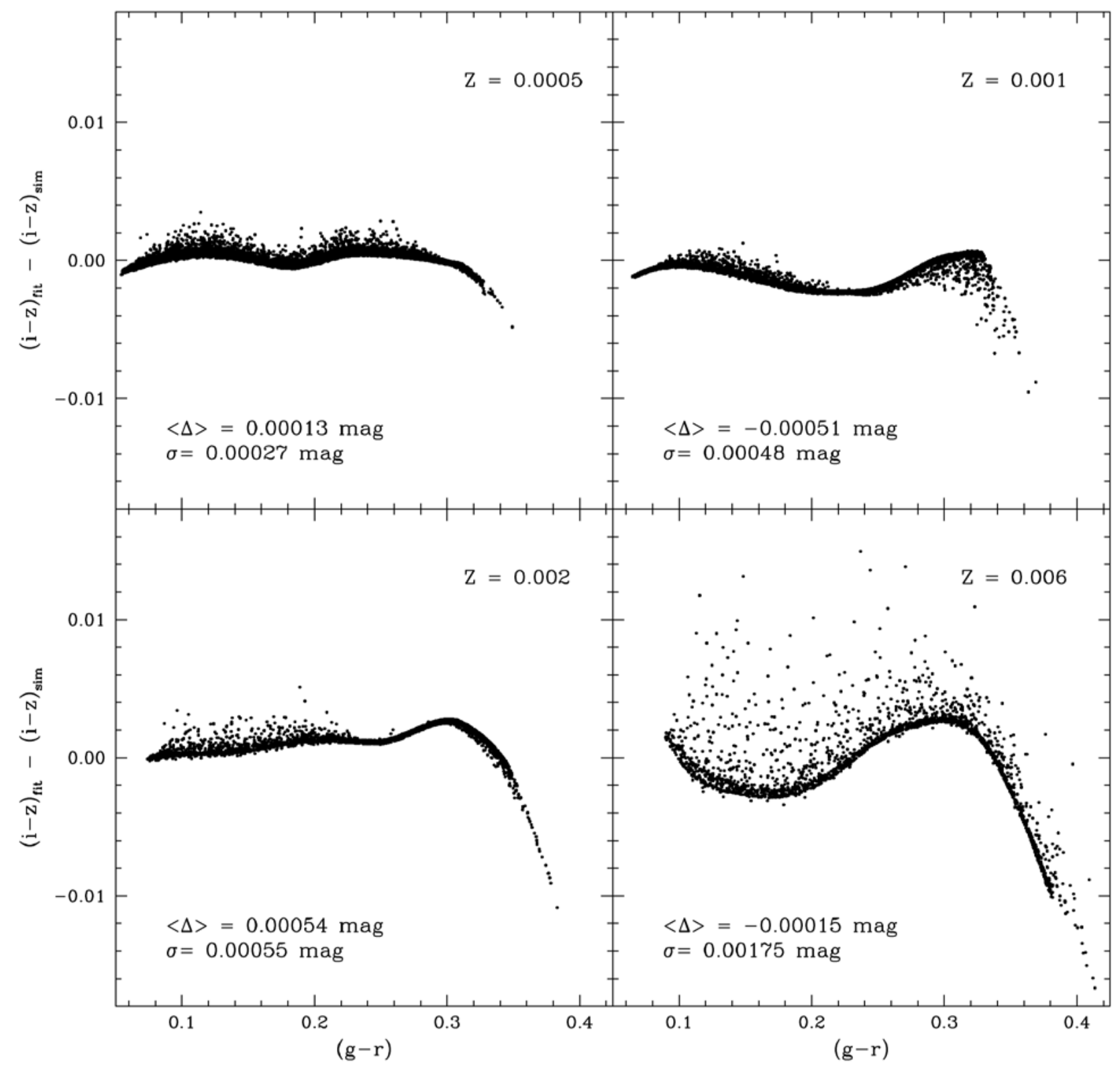

FIG. 7.- As in Figure 3 but for $(i-z)_{0}$.

Sweigart, A. V., \& Catelan, M. 1998, ApJ, 501, L63

van Albada, T. S., \& Baker, N. 1971, ApJ, 169, 311

Wilhelm, R., et al. 2008, preprint (astro-ph/0712.0776)

VandenBerg, D. A., Swenson, F. J., Rogers, F. J., Iglesias, C. A., \&

Alexander, D. R. 2000, ApJ, 532, 430 


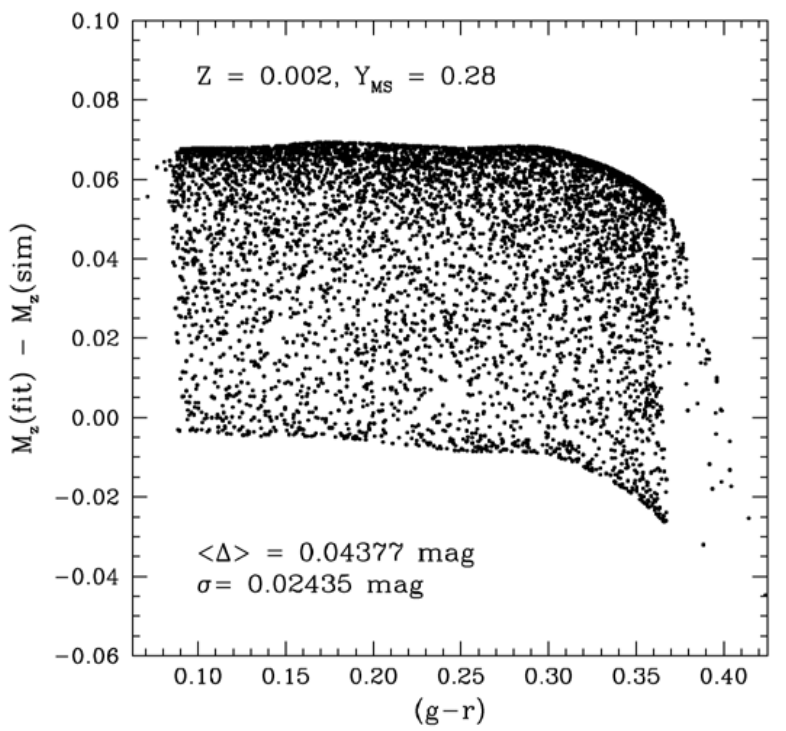

FIG. 8.- Effect of an increase in the helium abundance upon the derived PLpsC relation for $z$. Here we give the difference between the quantities predicted by equation $(3)$ and the input values from HB simulations computed for a $Y_{\mathrm{MS}}=0.28$ and $Z=0.002$, as a function of $(g-r)_{0}$. In this plot, 6000 randomly selected synthetic RR Lyrae stars are shown. 


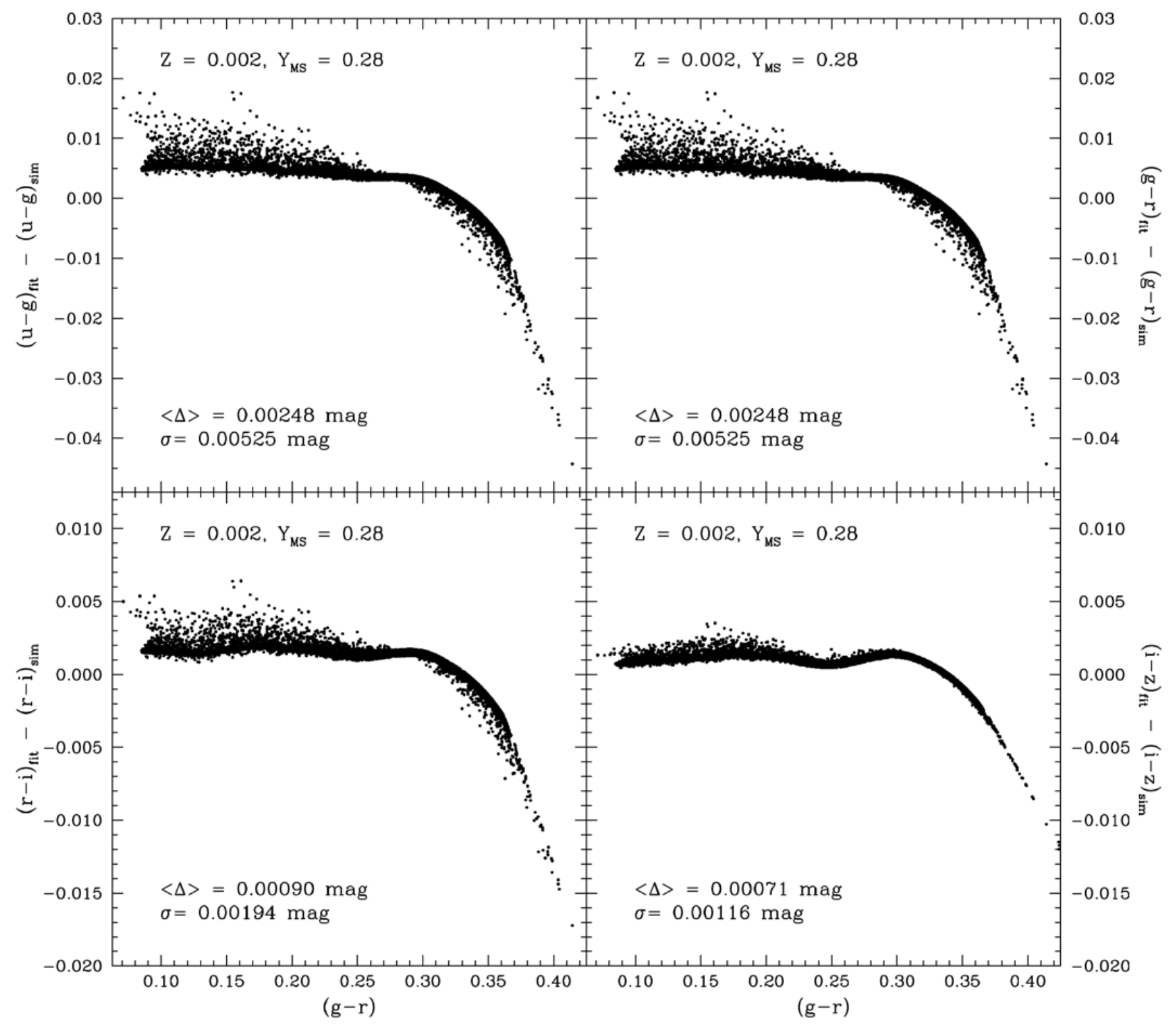

FIG. 9.- Effect of an increase in the helium abundance upon the derived relations for the $(u-z)_{0},(g-r)_{0},(r-i)_{0}$ and $(i-z)_{0}$ colors. Here we give the difference between the quantities predicted by equation 3 and the input value from HB simulations computed for a $Y_{\mathrm{MS}}=0.28$ and $Z=0.002$, as a function of $(g-r)_{0}$. In this plot, 6000 randomly selected synthetic RR Lyrae stars are shown. 